\title{
Géolinguistique
}

16 | 2016

Varia

\section{La seconde diphtongaison de 'ŏ latin dans le domaine occitan}

The Second Diphthongization of Latin 'ŏ in the Occitan Domain

Xavier Barillot, Sylvain Casagrande et Olivier Rizzolo

\section{(2) OpenEdition}

Journals

Édition électronique

URL : http://journals.openedition.org/geolinguistique/533

DOI : 10.4000/geolinguistique.533

ISSN : 2650-8176

Éditeur

UGA Éditions/Université Grenoble Alpes

\section{Édition imprimée}

Date de publication : 1 décembre 2016

Pagination : 161-179

ISBN : 978-2-84310-342-1

ISSN : 0761-9081

Référence électronique

Xavier Barillot, Sylvain Casagrande et Olivier Rizzolo, "La seconde diphtongaison de 'ŏ latin dans le domaine occitan », Géolinguistique [En ligne], 16 | 2016, mis en ligne le 15 février 2019, consulté le 01 novembre 2020. URL : http://journals.openedition.org/geolinguistique/533 ; DOI : https://doi.org/ 10.4000 /geolinguistique.533 


\title{
La seconde diphtongaison de 'ŏ latin dans le domaine occitan
}

\author{
Xavier Barillot \\ Sylvain Casagrande \\ Olivier Rizzolo \\ Université Nice-Sophia-Antipolis, \\ UMR 7320 : laboratoire Bases, Corpus, Langage
}

\section{Résumé}

Le but de cet article est de tenter de rendre compte de la diphtongaison de 'ŏ latin en [w'o] dans un certain nombre de parlers du sud de la France. Le caractère, mystérieux à différents égards, de ce processus n'a pas trouvé d'explication définitive dans la littérature. Nous essaierons de montrer que l'on peut faire appel à un seul mécanisme (rééquilibrage du système phonologique) agrémenté de mécanismes additionnels (partage de traits, $\mathrm{PCO}$ ), paramétrés différemment, en fonction des parlers, pour expliquer le processus examiné ici.

\section{Mots-clés}

Dialectologie, occitan, phonologie, diphtongaison.

\section{Abstract}

This paper aims at giving an account for the diphthongization of latin ' $\breve{o}$ to [w's] in a number of dialects in the South of France. The mysterious nature of this process has not found yet a definitive explanation in the literature. We will try to show that we can invoke a unique mechanism (rebalancing of the phonological system), completed with additional mechanisms (features sharing, OCP), configured differently according to the dialects, to explain the process at stake here. 


\section{Keywords}

Dialectology, Occitan, phonology, diphthongization.

\section{Point de départ : la seconde diphtongaison de 'ŏ latin dans les Alpes-Maritimes}

À partir des $\mathrm{XV}^{\mathrm{e}}$ et $\mathrm{XVI}^{\mathrm{e}}$ siècles (Gasiglia, 1984 : 26-29), les parlers des Alpes-Maritimes (dorénavant, $\mathrm{AM}$ ) ont diphtongué régulièrement les $O$ brefs toniques latins ('ŏ) en [w'o]. Dalbera (1994 : 328-338), après Schürr (1956 : 196-199), qualifie cette diphtongaison de «seconde diphtongaison», une série de 'ŏ ayant diphtongué, au préalable, en contexte métaphonique, d'abord devant une désinence flexionnelle $-\bar{\imath}$, POTUĪ $>$ [pw'Ek] je pus, puis devant une consonne palatale, NOCTE $>$ [nw'ejt] nuit ou vélaire, FOCU $>$ [fw' $\varepsilon \mathrm{k}]$ feu ou encore $\mathrm{v}$ final roman $[-\mathrm{w \#}]^{1}$. Si le conditionnement de cette première diphtongaison de 'ŏ n'est pas particulièrement mystérieux, et son résultat facilement repérable car contenant le plus souvent au moins un élément palatal, celui de la seconde diphtongaison est, lui, beaucoup plus énigmatique. Avant de voir en quoi ce conditionnement pose un défi, voici une illustration du phénomène en question sous (1) :

(1) Seconde diphtongaison de 'ŏ latin (Ronjat, 1930 : 159-163; Séguy, 1954 ; Dalbera, 1994 : 328-338; Schurr, 1956 : 196-199) :

$\begin{array}{llll}\text { PORCU } & > & {\text { [pw'ork }]^{2}} & \text { porc } \\ \text { SOMNU } & > & {[\text { sw'on }]^{\prime}} & \text { sommeil } \\ \text { DE FORAS } & > & {[\text { defw'oro }]} & \text { dehors }\end{array}$

1. Les faits sont évidemment plus complexes. Le lecteur intéressé par cette première diphtongaison est invité à consulter Dalbera (1994 : 317-327), Schürr (1956), Ronjat (1930 : 163-188) ou Anglade (1921 : 72-76).

Signalons que les dialectes occitans, et en particulier ceux des AM, n'ont pas subi comme le gallo-roman les $1^{\text {re }}$ et $2^{\mathrm{e}}$ diphtongaisons romanes des $3^{\mathrm{e}}$ et $4^{\mathrm{e}}$ siècles qui ont affecté les $e$ et $o$ brefs toniques latins en position libre (p. ex. OPERA > ['obra] et MEL $\left.>\left[\mathrm{m}^{\prime} \varepsilon l\right]\right)$. De plus, les dialectes des AM et de Provence orientale ont systématiquement fait régresser cette première diphtongaison de ' $\mathrm{o}$ devant -w\# selon Ronjat (1930 : 181-185) et Dalbera (1984 : 326).

2. Nous ne chercherons pas à donner, ici, une explication au fait que le premier exemple, ci-dessus, soit passé à travers les mailles du filet de la première diphtongaison malgré la présence de la syllabe finale-CU. Néanmoins, il convient de noter que quelques parlers (Esparron [05], Larche [04], etc.) montrent une réalisation du type [pq'eRk] ou [pu'œRk]. 
Il est important de noter que la seconde diphtongaison n'est imputable ni à un mécanisme métaphonique, ni à la présence d'une palatale ou d'une vélaire dans le voisinage, comme c'est le cas pour la première diphtongaison (car il n'existe plus de 'ŏ dans ces contextes). Ce qui confère son caractère mystérieux à ce changement.

Dalbera (1994 : 328-338), Schürr (1956 : 197) et Anglade (1921: 76) se sont interrogés sur le conditionnement de ce changement : ils notent que cette diphtongaison est observée plus systématiquement en syllabe fermée par une consonne alvéolaire $(r, l, n, s)$, i.e. en niçois, COSTA $>$ [kw' osta] côte, COLLU $>\left[\mathrm{kw}^{\prime} \mathrm{ol}\right] \mathrm{col}$, PONTE $>$ [pw' ont $]$ pont qu'en syllabe ouverte, i.e. * LONA > [1'ona] flaque.

Dalbera, qui ne parvient pas à capter la nature exacte de ce processus, conclut que les quelques réflexions qu'il livre (jeu de la morphologie, rééquilibrage du système, alignement sur la série antérieure...) ne représentent, dans son esprit, rien d'autre qu'une suggestion.

Nous nous proposons, dans cet article ${ }^{3}$, d'essayer d'y voir plus clair et de fournir une solution au mystère posé par la seconde diphtongaison de 'ŏ.

\section{Le conditionnement et l'aire d'extension du phénomène}

\subsection{Un conditionnement biscornu}

Pourquoi une diphtongaison aurait-elle lieu en syllabe fermée par une consonne alvéolaire? La question mérite d'être posée pour deux raisons : 1) les phonologues n'ont pas l'habitude de voir une voyelle diphtonguer en syllabe fermée, c'est le plus souvent en syllabe ouverte que ce type de processus vocalique est observé (comme c'est le cas pour la diphtongaison spontanée du gallo-roman : voir p. ex. Zink (1986: 53-56); et 2) il apparaît aux mêmes phonologues encore plus étrange de constater, qu'en plus d'un conditionnement structurel inhabituel, il faille ajouter une condition mélodique. En quoi une propriété mélodique d'une consonne, ici son alvéolarité, jouerait-elle un quelconque rôle dans une diphtongaison? L'accent, comme c'est le cas dans de très nombreuses langues, la présence d'un glide à la droite de la future diphtongue, comme on peut l'observer, entre autres, dans la diachronie du français (vECTURA > voiture), la combinaison de l'un et de l'autre de ces facteurs sont des vecteurs de changement attestés, fréquents et attendus. Le caractère alvéolaire d'une sonante $(r, l)$ ou d'une

3. Qui fait suite à une communication donnée à l'occasion de la conférence R(éseau) $\mathrm{F}$ (rançais de)P(honologie) 2015. 
obstruante $(s)$, non. Pourrait-il s'agir, dans le premier (syllabe fermée) comme dans le deuxième cas (alvéolarité de la consonne) d'une simple illusion d'optique?

En ce qui concerne la structure syllabique, les données pour les AM montrent une proportion comparable de contrevenants : $20 \%$ des ['o] en syllabe fermée et $25 \%$ des ['o] en syllabe ouverte ne diphtonguent pas ${ }^{4}$. Dans cette perspective, le caractère fermé de la syllabe ne semble pas être un vecteur de la diphtongaison. Cette première bizarrerie n'était qu'une vulgaire illusion.

En ce qui concerne le caractère alvéolaire de la consonne suivante, nous ne pouvons, à ce stade, que constater, sans pouvoir l'expliquer, tout comme (Dalbera, 1994), que la diphtongaison survient essentiellement devant une consonne alvéolaire. Nous reviendrons évidemment sur ce point, capital, plus tard.

Admettons pour l'instant, à contrecœur, que la diphtongaison de ['o] serait due à la nature alvéolaire de la consonne suivante (!).

\subsection{L'aire d'extension du phénomène}

Nous avons voulu vérifier l'aire d'extension exacte de ce phénomène et avons obtenu le résultat suivant ${ }^{5}$, reproduit sous (2) :

(2) Départements concernés par la seconde diphtongaison de ' $\mathrm{o}$ :

Alpes-de-Haute-Provence (04), Hautes-Alpes (05), Alpes-Maritimes (06), Ardèche (07), Aveyron (12), Bouches-du-Rhône (13), Cantal (15), Drôme (26), Gard (30), Haute-Loire (43), Lot (46), Lozère (48), Var (83), Vaucluse $(84)^{6}$.

Comme nous pouvons le voir sur la carte (assez schématique) ci-contre, le phénomène dépasse largement le cadre des AM.

4. Données issues de l'examen des dictionnaires de Castellana (2001) et de Calvino (1993).

5. Toutes les données sont issues du THESOC (base de données lexicales, en ligne, des parlers occitans, <http://thesaurus.unice.fr/>).

6. Nous avons aussi trouvé quelques cas de diphtongaisons dans les départements de l'Allier (03), de l'Aude (11), de la Corrèze (19), de la Creuse (23), de la Dordogne (24), de l'Isère (38), du Puy-de-Dôme (63), du Tarn-et-Garonne (82), de la HauteVienne (87). 


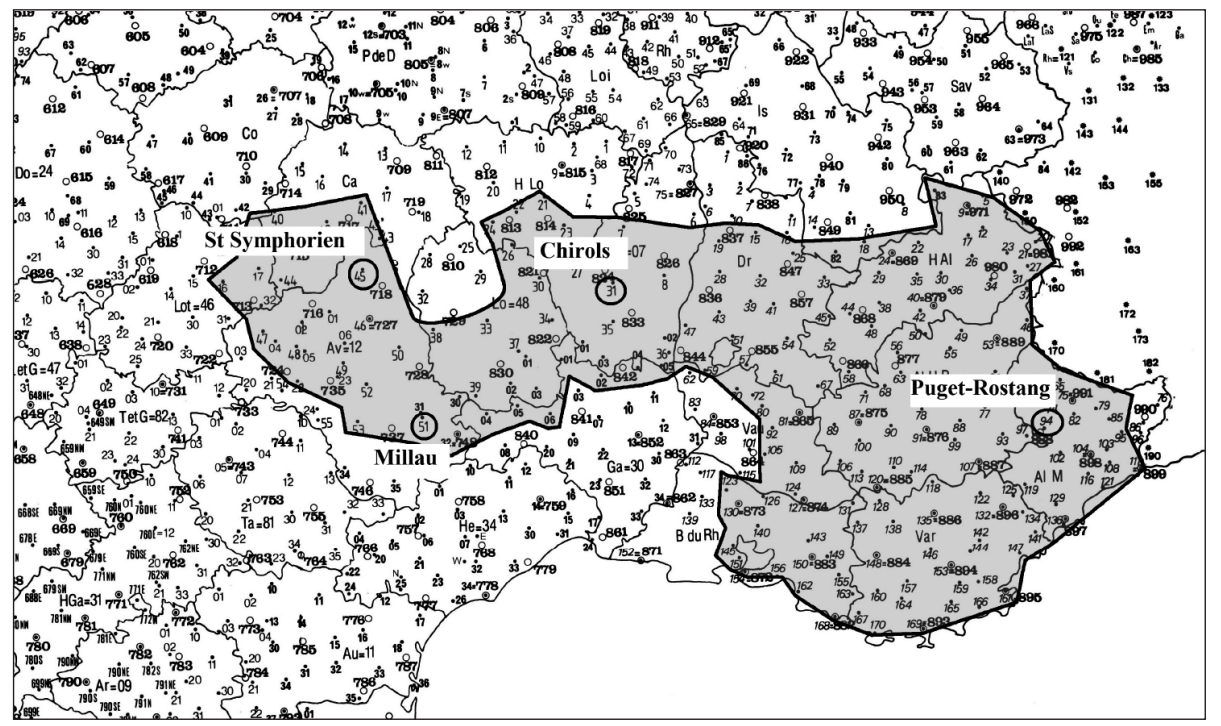

\section{Un conditionnement identique?}

La question qui vient immédiatement à l'esprit est la suivante : est-ce que ce phénomène connaît, dans les autres départements, un conditionnement identique à celui des $\mathrm{AM}$ ?

La réponse ne s'est pas trop fait attendre : nous avons pu observer, assez rapidement, que la diphtongaison se produisait dans un contexte assez différent de celui mis en évidence par Dalbera. Elle semblait parfois n'avoir lieu qu'après consonne labiale/vélaire/labio-dentale, comme on peut le voir sous (3) :

(3) Saint-Symphorien (Aveyron, localité $\mathrm{n}^{\circ} 45$ ).

\begin{tabular}{|c|c|c|}
\hline $\begin{array}{l}\text { /labiale___ } \\
\text { /labio-dentale__ } \\
\text { /vélaire__ }\end{array}$ & $\begin{array}{l}\text { [pw'orto], [bw'os], [mw'ol] } \\
\text { [fw'ol] } \\
{[\mathrm{kw} \text { 'osto], [gw'orp] }}\end{array}$ & $\begin{array}{l}\text { porte, forêt, mou } \\
\text { enragé } \\
\text { montée, corbeau }\end{array}$ \\
\hline utre consonne__ & $\begin{array}{l}{\left[\mathrm{r}^{\prime} \mathrm{ok}\right],\left[\mathrm{s}^{\prime} \text { ore }\right],[\mathrm{t} \text { 'orse }],} \\
{\left[\mathrm{l}^{\prime} \text { ogo }\right],[\text { lo n'ostro] }}\end{array}$ & $\begin{array}{l}\text { rocher, sæur, tordre, louée } \\
\text { (des domestiques), la nôtre }\end{array}$ \\
\hline
\end{tabular}

De plus, de manière intéressante, la diphtongaison a lieu indépendamment de la nature de la consonne suivante: [kw' op] fois, [kw' ofre] coffre à grains...

Du point de vue du phonologue théoricien, cette perspective est, de loin, plus satisfaisante : on comprend beaucoup mieux qu'une consonne puisse propager un trait/élément sur un segment adjacent, surtout lorsque 
cette dernière partage un/des trait(s) avec ce segment. Une consonne labiale/vélaire/labio-dentale partage, en effet, au moins un trait (labialité/ vélarité) avec [o] (voir p. ex. Bucci, 2013) pour une proposition similaire pour les dialectes des Pouilles). Dans cette perspective, le premier élément de la diphtongue [w' $\mathrm{w}$ ] représenterait une propagation du caractère labial/vélaire de la consonne précédente, constituant ainsi une sorte de renforcement.

Ainsi, sur le plan théorique, tout irait pour le mieux dans le meilleur des mondes : la diphtongaison de ' $\breve{o}$ latin représenterait un cas de renforcement par propagation d'un trait mélodique. Tout irait pour le mieux si cette première observation ne s'était pas, rapidement, trouvée ébranlée.

En effet, un examen un peu plus poussé des données révèle, presque tout aussi rapidement, que de nombreux parlers de l'aire concernée connaissent cette diphtongaison après n'importe quelle consonne(!). C'est le cas, notamment, pour le parler de Millau, comme on peut le voir sous (4) :

(4) Millau (Aveyron, localité $n^{0} 51$ ).

\begin{tabular}{|c|c|c|}
\hline /labiale & [pw'orto], [bw'os], [mw'ol] & porte, forêt, mou \\
\hline /labio-dentale__ & [fw'ol] & enragé \\
\hline /vélaire__ & [kw'osto], [gw'orp] & montée, corbeau \\
\hline tre consonne__ & $\begin{array}{l}{[\mathrm{rw} \text { 'ok], [sw' ore }],[\mathrm{tw} \text { 'orse }],} \\
{\left[\mathrm{lw}^{\prime} \text { ogo }\right],[\text { lo nw' ostro] }}\end{array}$ & $\begin{array}{l}\text { rocher, sæur, tordre, louée } \\
\text { (des domestiques), la nôtre }\end{array}$ \\
\hline
\end{tabular}

Malgré la contrariété évidente occasionnée par ces nouvelles données, il demeure un élément positif. La diphtongaison dans ces parlers se fait, ici aussi, indépendamment de la consonne suivante : fois [kw'op], neuve [nw'sbo]...

$\mathrm{Si}$, au contraire de ce que nous avions constaté au même titre que (Dalbera, 1994), la consonne suivante ne fait rien à l'affaire et si, au contraire de ce que nous avons, très momentanément suspecté, la consonne précédente ne fait rien non plus à l'affaire, qu'est-ce qui peut bien conditionner, alors, cette diphtongaison?

\section{4. À la recherche du conditionnement}

\subsection{Typologie}

Pour répondre à cette question, nous avons entrepris d'examiner tous les parlers qui sont concernés par cette diphtongaison. Nous avons décidé de passer en revue ces parlers indépendamment de tout critère statistique et avons, ainsi, considéré les parlers qui diphtonguent beaucoup comme ceux qui diphtonguent moins, voire peu. 
264 localités (chacune correspondant à un point d'enquête du THESOC) connaissent, visiblement, dans des proportions différentes, la diphtongaison de 'ŏ latin. Pour l'étude en question, nous avons examiné les parlers d'environ 110 d'entre elles.

Sur le plan de la collecte des données, nous avons exporté dans un fichier Excel 120000 questions/réponses d'enquêtes dialectologiques du THESOC et traqué, à l'aide de la fonction rechercher et des filtres-tri tous

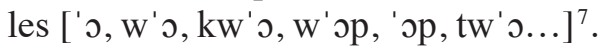

Voici, sous (5), un extrait de l'export :

(5) Export du THESOC :

\begin{tabular}{|c|c|c|c|c|c|c|c|c|}
\hline nép. & n'loc. & localité & dépt. & $n^{\circ}$ quest. & question & lemme & étymon latin & forme phonique \\
\hline 817315 & 741 & MILLAU & 12_AVEYRON & 2616 & corbeau & còrb & CŎRVUS & gw'orp \\
\hline 895656 & 741 & MILLAU & 12_AVEYRON & 8020 & porc & pòrc & PŎRCUS & pw'ork \\
\hline 817016 & 741 & MILLAU & 12_AVEYRON & 350 & araignée & aranha & ARANEUS & ir'one \\
\hline
\end{tabular}

Un examen, plus approfondi, des différents parlers nous a permis de mettre au jour deux nouveaux critères qui n'avaient pas été envisagés jusqu'ici. Nous avons, en effet, remarqué que, dans un certain nombre de parlers, la diphtongaison semblait bloquée lorsque la consonne suivante était une labiale/vélaire/labio-dentale (cinquième colonne du tableau sous (6), __ K/P/F). Dans un certain nombre d'autres, la diphtongaison semblait ne pas avoir lieu après un groupe obstruante-liquide (quatrième colonne du tableau sous (6), $\mathrm{C}=\mathrm{TR}$ ).

Les différents critères, qui se sont dégagés de cet examen, nous ont permis d'établir la typologie suivante sous (6) :

(6) Typologie :

\begin{tabular}{|c|c|c|c|c|}
\hline \multirow{3}{*}{ Types } & \multicolumn{4}{|c|}{ Contexte de la diphtongaison } \\
\hline & \multicolumn{3}{|c|}{$\mathrm{C}$} & \multirow{2}{*}{$\ldots$} \\
\hline & $\mathrm{C}=\mathrm{K} / \mathrm{P} / \mathrm{F}$ & Toute C & $\mathrm{C}=\mathrm{TR}$ & \\
\hline A & $\mathrm{x}$ & & $\mathrm{x}$ & $\mathrm{x}$ \\
\hline B & $\mathrm{x}$ & & $\mathrm{x}$ & \\
\hline C & $\mathrm{x}$ & & & $\mathrm{x}$ \\
\hline $\mathrm{D}$ & $\mathrm{x}$ & & & \\
\hline $\mathrm{E}$ & $\mathrm{x}$ & $\mathrm{x}$ & $\mathrm{x}$ & $\mathrm{x}$ \\
\hline F & $\mathrm{x}$ & $\mathrm{x}$ & $\mathrm{x}$ & \\
\hline $\mathrm{G}$ & $\mathrm{x}$ & $\mathrm{x}$ & & $\mathrm{x}$ \\
\hline $\mathrm{H}$ & $\mathrm{x}$ & $\mathrm{x}$ & & \\
\hline $\mathrm{I}$ & & & & \\
\hline
\end{tabular}

7. Dans certains parlers, la diphtongue peut prendre la forme [w'a/w'e], i.e. [sw'are] søur (Nice, AM), [kw'elo] colline (Saint-Maximin, Var)... Ce point, bien qu'intéressant, ne sera pas abordé ici. Dans notre étude, nous avons bien sûr pris en consi- 
La nature du contexte dans lequel se produit ou non la diphtongaison nous a donc permis de définir neuf types de dialectes (voir plus bas, pour la justification du nombre de types possibles). Le type A, p. ex. verrait le ' $\breve{~}$ latin diphtonguer en [w' $\mathrm{w}$ ] lorsque ce dernier suivrait une consonne labiale/ vélaire/labio-dentale. Le type $\mathrm{E}$, lui, diphtonguerait après n'importe quelle consonne.

Suivent sous (7) quelques exemples illustrant l'impossibilité/la possibilité de diphtonguer après un groupe obstruante-liquide/avant une consonne labiale/vélaire/labio-dentale :

(7) $\mathrm{TR} \&[\mathrm{~K} / \mathrm{P} / \mathrm{F}$ :

\begin{tabular}{|c|c|c|c|c|}
\hline & \multicolumn{2}{|c|}{$\mathrm{TR}=\mathrm{OK}$} & \multicolumn{2}{|c|}{$\mathrm{TR} \neq \mathrm{OK}$} \\
\hline Puget-Rostang, AM, loc no 94 & $\begin{array}{l}{\left[\text { frw' }^{\prime} 2^{\mathrm{nt}}\right]} \\
{[\mathrm{trw}} \\
\end{array}$ & $\begin{array}{l}\text { front } \\
\text { tonnerre }\end{array}$ & & \\
\hline Nice, $A M$, loc no 121 & & & $\begin{array}{l}{\left[\mathrm{fr}^{\prime} \text { วnte] }\right.} \\
{\left[\operatorname{tr}^{\prime} \tilde{\jmath}^{p}\right]}\end{array}$ & $\begin{array}{l}\text { front } \\
\text { tonnerre }\end{array}$ \\
\hline
\end{tabular}

\begin{tabular}{|c|c|c|c|}
\hline & \multicolumn{2}{|c|}{$\ldots \_\mathrm{K} / \mathrm{P} / \mathrm{F}=\mathrm{OK}$} & $\ldots$ \\
\hline Millau, Aveyron, loc $n^{\circ} 51$ & $\begin{array}{l}\text { [nw'obo] } \\
\text { [monw'sbro] }\end{array}$ & $\begin{array}{l}\text { neuve } \\
\text { manceuvre }\end{array}$ & \\
\hline Laurac, Ardèche, loc no 35 & & & \begin{tabular}{|ll} 
[n'ovo] & neuve \\
[mon'obro] & mancuvre
\end{tabular} \\
\hline
\end{tabular}

Bien entendu, les deux parlers qui ne connaissent pas la diphtongaison après un groupe obstruante-liquide $(\mathrm{TR} \neq \mathrm{OK})$ ou avant une consonne labiale/

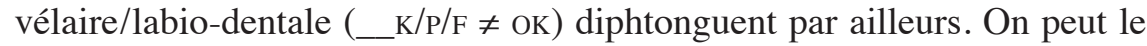
vérifier sous (8) :

(8) ' $\mathrm{o}>[\mathrm{w}$ ' $)$ par ailleurs :

\begin{tabular}{|c|c|c|}
\hline$\breve{o}>$ [w'o] & & \\
\hline Nice, $A M$, loc no 121 & $\begin{array}{l}\text { [pw' ork] } \\
\text { [sw' orba] }\end{array}$ & $\begin{array}{l}\text { porc } \\
\text { sorbe }\end{array}$ \\
\hline Laurac, Ardèche, loc no 35 & $\begin{array}{l}\text { [sw' ore] } \\
\text { [kw' osto] }\end{array}$ & $\begin{array}{l}\text { sour } \\
\text { versant }\end{array}$ \\
\hline
\end{tabular}

dération toutes les formes phoniques contenant les séquences [w'o], [w'a], [w'a], [w'p], [w'e], [w'c], [w'œ]..., au même titre que celles contenant la séquence [w'o]. 
En ce qui concerne le nombre de types prévus, étant donné qu'il y a trois paramètres :

1) consonne possible avant le site de diphtongaison (Toute $\mathrm{C}$ vs $\mathrm{C}=\mathrm{K} / \mathrm{P} / \mathrm{F})$

2) consonne simple vs groupe obstruante-liquide possible avant le site $(\mathrm{C}=\mathrm{TR})$;

3) diphtongaison bloquée ou non avant C labiale/vélaire/labio-dentale $\left({ }^{\mathrm{K}} / \mathrm{P} / \mathrm{F}\right)$.

On s'attend, logiquement, à 8 possibilités plus 1 (absence de diphtongaison), donc 9 types.

Sur les 9 types possibles, nous en avons, pour l'instant, trouvé 5. Voici, sous (9) la situation :

(9) Les 5 types mis au jour:

\begin{tabular}{|c|c|c|c|c|c|}
\hline \multirow{3}{*}{ Types } & \multicolumn{4}{|c|}{ Contexte de la diphtongaison } & \\
\hline & \multicolumn{3}{|c|}{$\mathrm{C}_{-}$} & \multirow{2}{*}{ K $\mathrm{K} / \mathrm{P} / \mathrm{F}$} & \\
\hline & $\mathrm{C}=\mathrm{K} / \mathrm{P} / \mathrm{F}$ & Toute $\mathrm{C}$ & $\mathrm{C}=\mathrm{TR}$ & & \\
\hline A & $\mathrm{x}$ & & $\mathrm{x}$ & $\mathrm{x}$ & \\
\hline B & $\mathrm{x}$ & & $\mathrm{x}$ & & \\
\hline $\mathrm{C}$ & $\mathrm{x}$ & & & $\mathrm{x}$ & Saint-Symphorien $(12,45)$ \\
\hline $\mathrm{D}$ & $\mathrm{x}$ & & & & \\
\hline $\mathrm{E}$ & $\mathrm{x}$ & $\mathrm{x}$ & $\mathrm{x}$ & $\mathrm{x}$ & Millau $(12,51)$ \\
\hline $\mathrm{F}$ & $\mathrm{x}$ & $\mathrm{x}$ & $\mathrm{x}$ & & Puget-Rostang $(06,94)$ \\
\hline $\mathrm{G}$ & $\mathrm{x}$ & $\mathrm{x}$ & & $\mathrm{x}$ & \\
\hline $\mathrm{H}$ & $\mathrm{x}$ & $\mathrm{x}$ & & & Chirols $(07,31)$ \\
\hline I & & & & & La Salvetat $(12,54)$ \\
\hline
\end{tabular}

\subsection{Hypothèses}

Étant donné les comportements, parfois très différents, des parlers en regard de la diphtongaison, il apparaît impossible de maintenir une seule et unique hypothèse. Il n'y aurait donc pas un mais deux conditionnements différents. Le même phénomène de surface renverrait, en réalité, à deux processus distincts. Ils apparaissent sous (10) :

(10) Les deux conditionnements en question :

1) un processus mélodique : $\mathrm{K} / \mathrm{P} / \mathrm{F}$ propagent sur la voyelle adjacente (partage de traits);

2) un processus structurel : 'o passe à w' o pour des raisons de rééquilibrage du système phonologique. 


\section{Conditionnement structurel}

Le conditionnement structurel serait illustré par Millau (tabl. (9), type E). La diphtongaison y est absolument générale, les consonnes précédant ou suivant le site n'ont aucune espèce d'impact : dans ce parler, tous les $o$ brefs accentués du latin ont diphtongué, comme le montrent les cas ci-dessous.

1) Après toute consonne ( $\left.\mathrm{C}_{-}\right)$:

[sw'ore] sœur [kw'osto] montée [pw'orto] porte [lw'ogo] louée (des domestiques)

[bw'os] forêt [gw'orp] corbeau [rw'ok] rocher [lobw'ostro] la vôtre [mw'ol] тои [fw'ol] enragé [tw'orse] tordre [lonw'ostro] la nôtre...

2) Avant consonne labiale/vélaire/labio-dentale (__K/P/F) :

$\begin{array}{ll}\text { [nw'sbo] } & \text { neuve } \\ \text { [monw'sbro] } & \text { manceuvre } \\ \text { [gorlw'spo] } & \text { varlope }\end{array}$

3) Après un groupe obstruante-liquide ( $\left.\mathrm{TR}_{-}\right)$:

$\begin{array}{ll}{\left[\text { trw' } 2^{\mathrm{n}}\right]} & \text { tonnerre } \\ \text { [grw' } \mathrm{gs}] & \text { gros } \\ \text { [prw' } \text { 'spe] } & \text { propre }\end{array}$

La question qui vient naturellement à l'esprit au vu de ces données est la suivante : quelle peut bien être la dynamique de ce changement?

Avant de tenter d'y répondre, voici, sous (11), un bref rappel des éléments connus pour le parler de Millau :

(11) Millau, éléments connus (contexte de la diphtongaison):

- derrière/devant toute $\mathrm{C}$

- sous l'accent

- seul ŏ connaît ce processus

Si ces seuls éléments ne nous donnent pas un éclairage suffisant, d'autres données, qui ont été occultées jusqu'à présent, peuvent venir compléter le tableau et permettre d'expliquer pourquoi tous les 'ŏ latins passeraient à [w' $)]$ dans ce type de parlers.

Il se trouve que, dans ce parler, certains A toniques et tous les A atones latins sont systématiquement passés à [0] (voir Anglade, 1921 : 48; 96-97; Ronjat, 1930 : 191-193, 209-210, 214, 290-291). C'est ce qu'on peut voir sous (12) : 
(12) $a>[0]:$

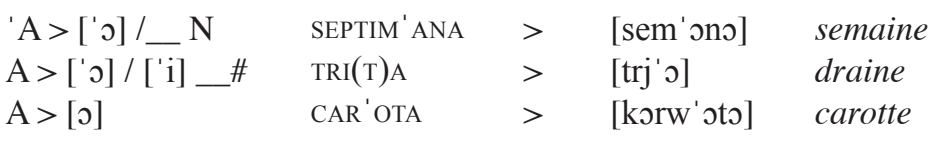

Ce dernier élément, capital, nous permet de poser que le passage de ['o] à [w'o] est possiblement dû à un rééquilibrage du système vocalique. Dans cette perspective, ['o] deviendrait [w'o] parce que [a] devient [o] et permettrait ainsi d'éviter la confusion [a/o]. On peut voir l'évolution (volontairement simplifiée) des voyelles latines en occitan sous (13):

(13) dynamique du changement :

latin

roman

occitan avt $2^{\mathrm{e}}$ dipht après $2^{\mathrm{e}}$ dipht

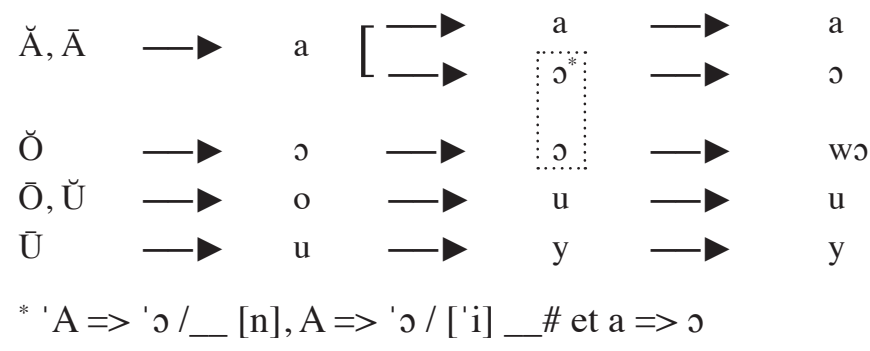

Ainsi, c'est parce que *banna donne [b'ono] que Bona donnerait [bw'ono], comme on peut l'observer, p. ex. dans le sud du Massif central (Lozère, Haute-Loire, Ardèche...).

Cette proposition, à savoir le rééquilibrage du système phonologique comme source de diphtongaison de 'ŏ latin, a pour mérite d'expliquer pourquoi seule cette voyelle est l'objet d'une diphtongaison.

\section{Conditionnement mélodique}

Ce conditionnement est illustré par la localité de Saint-Symphorien (voir tableau (9), type C). Dans le parler en question, la diphtongaison opère seulement après consonne labiale/vélaire/labio-dentale (K/P/F__ $)$, n'est jamais bloquée par une consonne labiale/vélaire/labio-dentale suivante $(\ldots \mathrm{K} / \mathrm{P} / \mathrm{F})$, est bloquée après un groupe obstruante-liquide (TR_), comme on peut le voir sous (14) : 
(14) Saint-Symphorien, conditionnement mélodique :

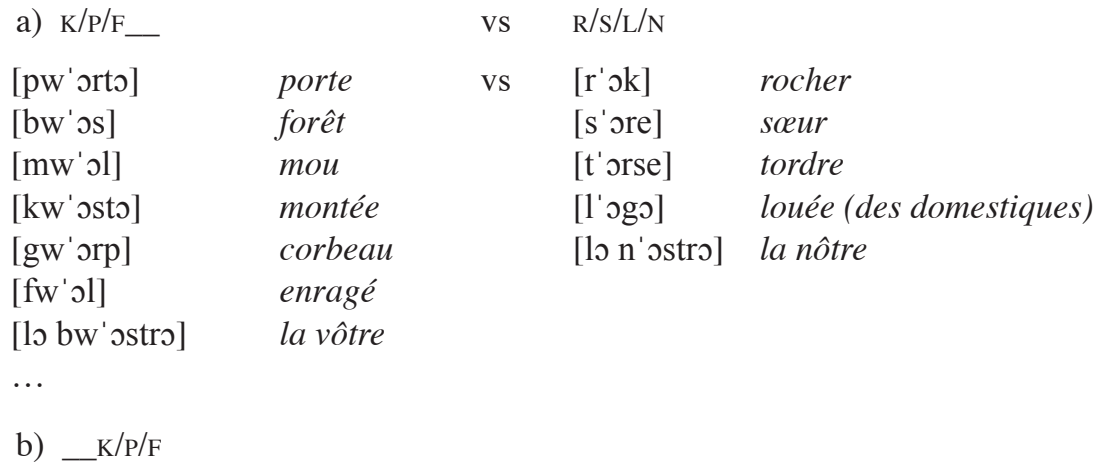

$\begin{array}{ll}{\left[\mathrm{kw}^{\prime} \mathrm{op}\right]} & \text { fois } \\ {\left[\mathrm{kw}^{\prime} \mathrm{ofre}\right]} & \text { coffre à grains } \\ {\left[\mathrm{mw}^{\prime} \mathrm{ople}\right]} & \text { meuble }\end{array}$

c) TR__ (attendu que la diphtongaison ne pourrait avoir lieu qu'après KR/PR/FR)

$\begin{array}{ll}\text { [gr'os] } & \text { gros } \\ \text { [eskl'ots] } & \text { sabot } \\ {[\mathrm{kl} \text { 'os] }} & \text { coquille } \\ \text { [fl'ot] } & \text { laine }\end{array}$

Quelle peut être la dynamique de ce conditionnement mélodique? Afin de répondre à cette question, considérons (voir (15)), dans un premier temps, les éléments connus à notre disposition.

(15) Saint-Symphorien, éléments connus (contexte de la diphtongaison) :

- seulement après $\mathrm{K} / \mathrm{P} / \mathrm{F}$

- sous l'accent

- seul 'ŏ connaît ce processus

Il est très tentant de penser que le fait que K/P/F et [o] partagent un trait de labialité/vélarité ne relève pas du pur hasard. Si tel est le cas, il s'ensuit que : 1) cette conjonction doit (suffire à) expliquer ce phénomène; 2) on peut s'attendre, sur le plan théorique, à observer un phénomène semblable pour les séquences $\mathrm{K} / \mathrm{P} / \mathrm{F}+[\mathrm{u} / \mathrm{y}]$ mais pas pour $\mathrm{K} / \mathrm{P} / \mathrm{F}+[\mathrm{e} / \mathrm{a} / \mathrm{i}]$.

C'est effectivement le cas pour $\mathrm{K} / \mathrm{P} / \mathrm{F}+[\mathrm{e} / \mathrm{a} / \mathrm{i}]$ : B' ${ }^{\prime}$ ELLA $>\left[\mathrm{b}^{\prime} \mathrm{\varepsilon l}\right.$ b] belle, $\mathrm{P}^{\prime}$ ASTOR $>$ [p'astre $]$ berger, FILIUS $>\left[\mathrm{f}^{\prime}\right.$ il $]$ fils...

Ce n'est pas le cas pour K/P/F + [u/y] : B' ŭCCA > [b'uko] bouche.

Seul [o] est concerné par la diphtongaison. Le partage de traits mélodiques ne semble donc pas être un bon candidat pour expliquer le condi- 
tionnement de la diphtongaison dans les parlers du type de celui de SaintSymphorien. Il faut donc rechercher une autre explication.

Une explication à moindre coût consisterait à supposer que, étant donné qu'à Saint-Symphorien, A tonique et A atone deviennent [0] comme à Millau, le parler de Saint-Symphorien doit, lui aussi, rééquilibrer son système. C'est la raison pour laquelle il connaitrait une diphtongaison. La différence avec le parler de Millau résiderait dans le fait que la diphtongaison n'est ici possible qu'après $\mathrm{K} / \mathrm{P} / \mathrm{F}$.

Dans cette perspective, on se trouverait dans un cas de diphtongaison (d'origine structurelle) suspendue à une condition de partage de trait (voir p. ex. Bucci, 2013) pour une illustration du partage de traits dans les dialectes des Pouilles). Ce partage de traits est illustré sous (16).

(16) Représentation de [bw'os] forêt vs [t'orse] tordre (éléments repris de Backley, 2011).

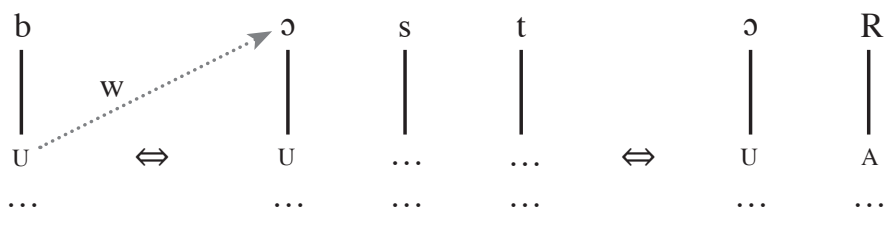

Dans la vision de Backley (2011) (suivant la voie tracée par Kaye et al., 1985), entre autres), les segments sont composés d'unités plus petites appelées éléments. Sans rentrer dans les détails de cette machinerie complexe, il suffit de dire que les consonnes labiales, les consonnes vélaires et les voyelles postérieures arrondies contiennent toutes l'élément U. Au contraire, notamment, des consonnes coronales, comme [t], pour lesquelles cet élément n'entre pas dans la composition.

La relation contractée ici entre [b] et [o] qui contiennent tous deux l'élément u permet à la consonne bilabiale de propager son trait labial sur [0] donnant ainsi naissance à une diphtongue.

Ainsi, la diphtongaison est possible pour [bw'os] forêt parce que [b] et [0] partagent un élément mais impossible pour [ $\mathrm{t}$ 'orse] tordre car [t] et [0] ne partagent aucun élément.

Signalons de plus que nous ne sommes pas les premiers à proposer une interaction entre la voyelle [0] et la consonne qui précède : Jean Séguy (1954), dans un article consacré à la diphtongaison de $[\varepsilon]$ et [0], remarque, alors que c'est inattendu, des débuts de diphtongaison pour ces deux voyelles en Ariège. Concernant [0], il précise que «l'initium labio-vélaire $w$ n'apparaît qu'après les consonnes labiales ou vélaires » et «qu'il est vraisemblable que les choses ont dû se passer ainsi pour la 
diphtongaison récente de $\grave{o}$ dans une grande partie du domaine occitan» (1954: 311).

Enfin, le partage de traits et donc la diphtongaison ne sont pas possibles après un TR, car un segment et donc un élément interviennent entre $\mathrm{K} / \mathrm{P} / \mathrm{F}$ et le [॰] suivant.

\section{Conditionnement « ni l'un ni l'autre» (?)}

Ce conditionnement «ni l'un ni l'autre» serait représenté par Puget-Rostang et Chirols (tableau (9), types F et H). Pour ces parlers, la diphtongaison est générale, elle est bloquée avant $\mathrm{K} / \mathrm{P} / \mathrm{F}$ et effective (Puget-Rostang) ou non (Chirols) après TR. Voici, sous (17) un aperçu de la situation.

(17) Puget-Rostang et Chirols : conditionnement «ni l'un ni l'autre» :

a) Toute C__ (Puget-Rostang)

[pw'oRk] porc [bw'osk] forêt [fw'ol] fou [kw'ola] colline [gw'orbo] corbeille [sw'ore] saur [tw'orse] tordre [rw' $\widehat{\mathrm{t}}[\mathrm{\jmath}]$ rocher [nw'oj] Noix [lw'ono] Flaque ...

b) _ $\mathrm{K} / \mathrm{P} / \mathrm{F}$ (Puget-Rostang)

[m'oble] outil

[gal'ofre] ceillet

[n'ovo] neuve

[t'ok] morceau

c) $\mathrm{TR}$

Puget-Rostang Chirols

[tRw'ono] tonner [tr'ono] tonner

[krw'os] tombe [kr's] paume

[sybrw'os] cal [gr'os] gros

[frw' $\left.2^{\mathrm{yt}}\right]$ front $[\mathrm{kl}$ 'o] terrasse

de culture

Pour les parlers de ces deux localités, il n'est pas envisageable de convoquer l'hypothèse mélodique étant donné que la diphtongaison a lieu après toute consonne.

L'hypothèse structurelle n'est pas viable non plus car, dans les AM, [a] tonique reste toujours [a] et [a] atone ne passe à [0] qu'en position finale, de plus de façon très partielle.

Si ni l'une, ni l'autre des hypothèses n'est envisageable, comment peuton rendre compte de la situation dans ce type de parlers? 
L'hypothèse la moins coûteuse consiste néanmoins à proposer que les parlers de ce type $(\mathrm{F}$ et $\mathrm{H})$ répondent, malgré les apparences (passage très partiel de [a] à [0]), à un rééquilibrage du système : [a] aurait commencé à passer à [0], poussant [0] à devenir [wo], mais le processus [a] $>[0]$ ne se serait pas poursuivi, ce sans rétractation de [wo] vers [0].

Des commentaires de Ronjat peuvent permettre, en partie, de soutenir cette hypothèse. Il note par exemple, pour le parler d'Aiguilles (05), que [a] tonique passe fréquemment à [0] devant nasale (Ronjat, 1930 : 191) et que la réalisation [0] de [a] atone aurait été plus importante dans les siècles passés dans les AM (Ronjat, 1930 : 208-209).

Dans ce sens, on peut mentionner une série d'indices épars dans les localités de l'arrière-pays niçois. Par exemple à l'Escarène, on peut entendre

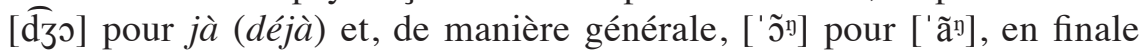
absolue, comme dans [m' $\tilde{\jmath}^{\eta}$ ], [p' ${ }^{\eta}{ }^{\eta}$ pour man (main) et pan (pain). De la même manière, on peut trouver dans les écrits de Sully Mainart (2001), écrivain dialectal de la vallée de la Tinée, de nombreuses attestations de cette vélarisation de [a] : Boirouos (Bairols) (village de S. Mainart), oquelos (celles-là), gronjo (grange), onoras (vous irez). On peut entendre également à Nice, acò de < A CASA DE (chez) ou [m'oko] < (e)m(bé) acò (avec ça, donc, alors).

Les parlers «ni l'un ni l'autre» seraient, dans cette perspective, des parlers répondant à un rééquilibrage du système. Cependant, les vestiges de ce rééquilibrage (passage de A tonique et atone à [0] dans certains contextes) ne seraient plus visibles dans les parlers actuels.

\section{Puget-Rostang et Chirols, une contrainte supplémentaire}

Une nouvelle contrainte, ignorée par les parlers examinés jusqu'à présent mais connue par les parlers de Puget-Rostang et Chirols, demande maintenant à être expliquée. On peut en voir une illustration sous (17b). Dans ces deux parlers, la diphtongaison est bloquée lorsque la voyelle cible survient devant une consonne labiale/vélaire/labio-dentale, comme c'est le cas, par exemple, pour [m'sble] outil, *[mw'sble].

L'arsenal phonologique possède, heureusement, un outil qui permet de rendre compte de ce genre d'impossibilités dans les langues : le principe du contour obligatoire ou PCO. Ce dernier dit que deux segments adjacents ne peuvent être identiques ou trop semblables ${ }^{8}$, ce qui est le cas ici. En effet,

8. Le PCO a d'abord été proposé pour rendre compte de dissimilations de tons dans les langues tonales. Il a été, par la suite, exporté pour rendre compte des contraintes 
[0] ne pourrait pas passer à [wo] devant une consonne labiale/vélaire/ labio-dentale, car [w] leur est trop semblable d'un point de vue mélodique. Comme on peut le voir sous (18), un mot comme [kw'op] n'est pas possible et doit donc être réalisé comme [k'op] parce que [w] et [p] partagent le trait labial (ou, selon la terminologie de Backley, l'élément U).

(18) PCO :

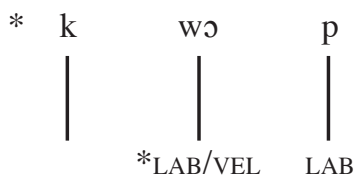

Cette explication, bien que permettant de rendre compte, d'une manière économique, de l'impossibilité pour [o] de diphtonguer devant une consonne labiale/vélaire/labio-dentale dans ces parlers, pose, malheureusement, au moins deux problèmes :

1) si [w'o] est une vraie diphtongue et que [w] fait ainsi partie du noyau, cela implique que le PCO est ici transcatégoriel, i.e. il pourrait s'appliquer entre une consonne et une voyelle. Ceci ne serait pas particulièrement problématique au regard de la théorie phonologique et des données qui viennent la conforter (on peut observer la chose en arabe classique). Cependant, un PCO transcatégoriel aurait une implication fâcheuse ici. En effet, s'il s'agissait bien d'un mécanisme de ce type, on ne devrait pas pouvoir observer des configurations du type [əp, ək, of...] dans lesquelles $\curvearrowright$ et K/P/F partagent aussi le trait labial ou vélaire. Pourtant elles sont évidemment nombreuses dans ces parlers...

2) si [w'o] n'est pas une vraie diphtongue et que [w] représente un élément consonantique, on ne comprend pas pourquoi la «diphtongaison» n'aurait lieu que sous l'accent : la nécessité de ce dernier semble indiquer qu'il s'agit bien d'un processus nucléaire et non d'un processus consonantique.

Nous nous retrouvons ici dans une impasse. Pourtant, malgré cette double problématique, il semble bien qu'on a affaire à une vraie contrainte de type PCo. Pour preuve, tous les parlers occitans (même ceux de Saint-

relatives aux cooccurrences de segments, notamment dans les langues sémitiques (voir p. ex. McCarthy, 1986). 
Symphorien et Millau) connaissent la même impossibilité : on n'observe jamais [w' $]$ devant un [w] suivant, comme on peut le voir sous (19):

(19) $*\left[w^{\prime} \jmath w\right]:$

Saint-Symphorien (diphtongue seulement après K/P/F, _K $/ \mathrm{P} / \mathrm{F}=0 K$ ) : [kw' $\mathrm{pp}]$ fois mais [n'ow] neuf (numéral), [m'owo] meule...

Millau (diphtongaison générale, $\_\mathrm{K} / \mathrm{P} / \mathrm{F}=$ оK) : [nw'obo] neuve mais [n'əw] neuf (numéral), ['ow] ouf...

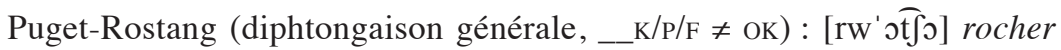
mais [m'oble] outil et bien sûr [n'ow] neuf (numéral), [k'ow] fois...

Chirols (diphtongaison générale, __ K/P/F $\neq$ ок) : [w'or] jardin et bien sûr [n'əw] neuf (numéral), [p' əw] peur...

Ceci semble indiquer que les parlers, dans leur ensemble, ne tolèrent pas les consécutions (adjacence non stricte) du type *ww.

Si ces parlers, comme nous le pensons, répondent bien à une contrainte de type $\mathrm{PCO}$, il faudrait pouvoir répondre à une autre question : pourquoi le PCO ne serait effectif que lorsque la consonne incriminée se trouve à droite de la voyelle et pas lorsqu'elle se trouve à gauche de cette dernière, i.e. [kw' ola] colline vs [t'ok] morceau (Puget-Rostang)?

Nous n'avons pas, malheureusement, dans l'état actuel des choses, de réponse à fournir.

\section{Conclusion}

Nous avons essayé de montrer dans cet article que l'origine de la diphtongaison de [0] dans les parlers occitans pouvait avoir une origine unique, à savoir un rééquilibrage du système phonologique. Les différences observées (les différents types identifiés jusqu'à présent, voir tableau (9)) ne représenteraient que des paramétrages différents propres à chaque parler/ ensemble de parlers : partage de traits pour Saint-Symphorien, PCO pour la Provence.

Notre proposition aurait, de plus, l'avantage d'être conciliable avec l'observation de Dalbera (1994) relative au caractère alvéolaire de la consonne suivant le site de diphtongaison. L'auteur aurait effectivement raison : on n'observe la diphtongaison qu'avant consonne alvéolaire (du moins, en Provence). Mais notre hypothèse permettrait d'expliquer ce qui semble être une aberration. En quoi, en effet, une propriété mélodique donnée, ici le caractère alvéolaire de la consonne, favoriserait-elle une diphtongaison? Il ne s'agirait là que d'une illusion d'optique : ce n'est 
pas que [kw'osto] côte ou [pw'ont] pont sont possibles parce que [o] précède une alvéolaire mais, plutôt, que *[kw'sp] est impossible en raison du caractère vélaire/labial/labio-dental de la C suivante (PCO).

Un certain nombre de points méritent encore d'être expliqués. Parmi ceux-ci, la question du domaine du PCO. Il faudrait, aussi, pouvoir répondre à la question de savoir pourquoi la diphtongaison est tantôt possible (PugetRostang) tantôt impossible (Chirols) après un groupe obstruante-liquide (TR_). Est-ce que les quatre types logiquement possibles restant existent bien ou non? Et s'ils n'existent pas, comment peut-on l'expliquer? Un certain nombre de questions restent donc encore en suspens même si notre analyse permet de rendre compte de manière unifiée de la diphtongaison de 'ŏ latin dans les parlers du sud de la France.

Un dernier point qui, nous le pensons, mérite une attention particulière : l'aire observée ne correspond à rien de connu, ce à tout point de vue (administratif, historique, géographique...). En particulier, elle transcende une partition dialectale bien établie par ailleurs (provençal, alpin, languedocien...). La perspective d'une origine commune au phénomène, l'origine structurelle, contrariée par un ensemble de paramètres propres à chaque parler (PCO, partage de traits et d'autres à expliquer/découvrir) permettrait de mieux appréhender l'étrangeté de l'aire examinée.

\section{RÉFÉRENCES BIBLIOGRAPHIQUES}

Atlas linguistiques de la France par région: ALAL, ALMC, ALP, ALLOR, ALLOC et ALG, CNRS. THESOC en ligne : <http://thesaurus.unice.fr/>.

Anglade Joseph, 1921, Grammaire de l'ancien provençal ou ancienne langue d'oc. Phonétique et morphologie, Paris, Klincksieck.

BACKLEy Phillip, 2011, An Introduction to Element Theory, Edimbourg University Press.

Bucci Jonathan, 2013, Raddoppiamento sintattico induit par l'accent et réduction vocalique en Italie : perspectives phonologique et dialectologique, thèse de doctorat en linguistique, Université Nice-Sophia-Antipolis.

Castellana Georges, 2001, Dictionnaire niçois-français [1947], Nice, Serre.

CALvino Jean-Baptiste, 1993, Dictionnaire niçois-français, français-niçois [1905], Nîmes, Lacour.

DALBERA Jean-Philippe, 1994, Les parlers des Alpes-Maritimes, étude comparative, essai de reconstruction, Egham, Association internationale d'études occitanes.

Gasiglia Rémi, 1984, Grammaire du Nissart : essai de description d'un dialecte d'oc, Institut d'études niçoises.

KaYe Jonathan, Lowenstamm Jean \& Vergnaud Jean-Roger, 1985, «The Internal Structure of Phonological Representations: A Theory of Charm and Government», Phonology Yearbook, nº 2, p. 305-328. 
Mainart Sully, 2001, Crounicoi de Santo-Peirounello, Nice, Serre.

McCARThy John J., 1986, «OCP Effects: Gemination and Antigemination », Linguistic Inquiry, $\mathrm{n}^{\circ} 17$, p. 207-263.

Ronjat Jules, 1930, Grammaire Istorique des parlers provençaux modernes, t. 1, Montpellier, Société des langues romanes.

SCHÜRR Friedrich, 1956, «La diphtongaison romane», Revue de linguistique romane, $\mathrm{n}^{\circ} 20$, p. 107-144 et p. 161-248.

SÉGuY Jean, 1954, «À propos de la diphtongaison de è et ò ouverts », Annales $d u$ Midi : revue archéologique, historique et philologique de la France méridionale, tome 66, $\mathrm{n}^{\circ} 27, \mathrm{p} .307-311$.

ZINK Gaston, 1986, Phonétique historique du français, Paris, PUF. 